\title{
Dendritic Cell-Epithelium Interplay Is a Determinant Factor for Corneal Epithelial Wound Repair
}

\author{
Nan Gao, ${ }^{*}$ Jia Yin, ${ }^{*}$ Gi Sang Yoon, * \\ Qing-Sheng $\mathrm{Mi}{ }^{\dagger \neq \S}$ and Fu-Shin X. Yu* \\ From the Departments of Ophthalmology and Anatomy and Cell \\ Biology,* Wayne State University School of Medicine, Detroit; and \\ the Henry Ford Immunology Program ${ }^{\dagger}$ and the Departments of \\ Dermatology $y^{\ddagger}$ and Internal Medicine, ${ }^{\S}$ Henry Ford Health System, \\ Detroit, Michigan
}

The functions of intraepithelial dendritic cells (DCs) are critical for mucosal innate and adaptive immunity, but little is known about the role of tissue-specific DCs in epithelial homeostasis and tissue repair. By using the epithelial debridement wound model and CD11c-diphtheria toxin receptor mice that express a CD11c promoter-driven diphtheria toxin receptor, we showed that DCs migrate along with the epithelial sheet to cover the wound and that local depletion of DCs resulted in a significant delay in epithelial wound closure. In response to wounding, migratory epithelia produce CXCL10, thymic stromal lymphopoietin, and IL-1 $\beta$ and its antagonist soluble IL-1 receptor antagonist (sIL-1Ra); depletion of corneal DCs reversed their elevated expressions to a different extent, suggesting a DC-mediated positive feedback loop in epithelial gene expression. Furthermore, both CXCL10 and thymic stromal lymphopoietin were localized in migratory epithelia, suggesting that epithelial cells play a key role in DC infiltration and activation in injured corneas. On the other hand, DC depletion resulted in suppressed epithelial AKT activation, increased cell apoptosis, and decreased polymorphonuclear leukocyte infiltration in the healing cornea. These results indicate that DCs and epithelium form a functional entity at mucosal surfaces for maintaining corneal homeostasis and for tissue repair. (Am J Pathol 2011, 179:2243-2253; DOI: 10.1016/j.ajpath.2011.07.050)

Dendritic cells (DCs) are diverse and specialized hematopoietic cells that serve as an essential bridge between innate and adaptive immunity. ${ }^{1,2}$ DCs are categorized as conventional or myeloid DCs and plasmacytoid DCs, a rare population of circulating cells. Conventional DCs line the tissues of the body exposed to the exterior environment, such as the skin and the epithelia of the lung, ${ }^{3}$ the gut $^{4}$ and the cornea, ${ }^{5}$ where they survey tissues for incoming pathogens or the emergence of pro-inflammatory stimuli. $^{6}$ In the cornea, it is increasingly clear that, although macrophages only occupy the posterior stroma, DCs reside in both the stroma and the epithelium, both with phenotypically different subtypes. ${ }^{7-10}$ In corneal epithelium stratified with five to seven layers of cells, DCs residing at the basal epithelial layer are more numerous in the peripheral than in the central cornea. ${ }^{11}$ Some of the DCs at the central cornea insert processes between epithelial cells, and these processes might sample antigens from the environment. ${ }^{11-13}$ Emerging studies ${ }^{14,15}$ indicated a critical role for DCs in ocular inflammation and infection, especially HIV-1 keratitis. Two recent studies ${ }^{11,13}$ using epithelial point-injury and pro-inflammatory cytokines as stimuli reveal that DCs responded to these stimuli by changing cell orientation or migrating toward the site of stimulation. Although a role in maintaining epithelial homeostasis has been suggested, whether DCs are involved in epithelial wound healing and tissue repair has not been studied.

The avascular cornea consists of three layers: stratified epithelium, stroma, and endothelium. It has two specialized functions: forming a protective barrier and serving as the main refractive element of the visual system. The corneal epithelium, like other mucosa linings in the human body, is continuously subjected to physical, chemical, and biological insults, often resulting in a wound and loss of barrier functions. ${ }^{16-18}$ The corneal epithelium responds rapidly to injury, healing a wound by migrating as a sheet to cover the defect and to re-establish its barrier function. ${ }^{19-21}$ Prolonged corneal epithelial defects due to a delay in wound healing may result in sight-threatening

Supported by grants from the $\mathrm{NIH} /$ National Eye Institute [NEl; R01EY10869; EY13431; R21AR059976 to Q.-S.M.; EY17960 to F.-S.X.Y.; p30 EY04078 (NEI core to Wayne State University)] and Research to Prevent Blindness (Kresge Eye Institute).

Accepted for publication July 19, 2011

Address reprint requests to Fu-Shin X. Yu, Ph.D., Kresge Eye Institute, Wayne State University School of Medicine, 4717 St Antoine Blvd., Detroit, Ml 48201. E-mail: fyu@med.wayne.edu. 
complications, including corneal opacity, neovascularization, and microbial keratitis. ${ }^{22,23}$ In the wounded cornea, the epithelium plays a central role, as a key cell type in repairing the cornea and as the source of several growth factors. ${ }^{19,20}$ In addition to the epithelial cells, innate immune cells, such as polymorphonuclear leukocytes (PMNs) and $\gamma \delta \mathrm{T}$ cells, have also been involved in the regulation of epithelial wound healing in vivo. ${ }^{24-26}$ DCs are another cell population located at the basal layer of the corneal epithelium. ${ }^{12,27-30}$ Unlike PMNs and $\gamma \delta T$ cells, which are usually not found in the cornea, ${ }^{25,31}$ DCs are present and embedded in the epithelia of many mucosal surfaces. ${ }^{1,5}$ Thus, both epithelial cells and intraepithelial DCs are in the front line facing external environments and can sense danger signals, including tissue injury and infection. It is likely that the epithelial cells and DCs will interact with each other to form a coordinated action against adverse challenges. ${ }^{1}$ How these two types of residential cells interact and what roles DCs might play in mediating epithelial migration, proliferation, and wound healing in injured tissue remain largely unclear. Therefore, we proposed that DCs serve as an essential bridge between innate and adaptive immunity and between mucosal epithelial cells and innate effector cells in response to wounding and invading pathogens, resulting in a rapid epithelial wound healing and fastacting local innate response to eliminate infected cells and invading pathogens.

In this study, we took advantage of B6.DTR/enhanced green fluorescence protein (EGFP) mice that express a CD11c promoter-driven diphtheria toxin (DT) receptor (DTR) and EGFP ${ }^{32,33}$ and assessed the role of DCs in the regulation of corneal epithelial wound healing. ${ }^{31,32}$ Herein, we report that the lack of DCs in the cornea altered the epithelial response to injury, increased cell death, and impaired epithelial wound healing. By using the corneal epithelial wound model, we made several important discoveries applicable to other tissues, such as the skin and airway, and to other pathological conditions, such as delayed wound healing in diabetic skin and cornea.

\section{Materials and Methods}

\section{Mice}

Wild-type (WT) C57BL6 (B6) mice (age, 8 weeks; weight, 20 to $24 \mathrm{~g}$ ) and CD11c-DTR [B6.FVB-Tg (Itgax-DTR/ EGFP) 57Lan/J] mouse breeding pairs were obtained from Jackson Laboratory (Bar Harbor, ME). B6.CD11cDTR mice carry a transgene encoded for a simian DTREGFP fusion protein under the control of the murine CD11c promoter, which makes them sensitive to DC depletion with DT. B6-DTR mice were bred in-house, and their pups were subjected to genotyping before use. Animals were treated in compliance with the Association for Research in Vision and Ophthalmology Statement on the Use of Animals in Ophthalmic and Vision Research. The Institutional Animal Care and Use Committee of
Wayne State University, Detroit, MI, approved all animal procedures.

\section{Depletion of DCs}

B6-DTR mice were depleted of their DCs using either 50 ng of DT in $5 \mu \mathrm{L}$ of PBS administered subconjunctivally (s.c.) or $100 \mathrm{ng}$ of DT in $100 \mu \mathrm{L}$ of PBS administered i.p. 24 hours before wounding. ${ }^{14}$ The controls include B6DTR mice s.c. injected with the same amount of PBS or WT B6 mice injected with $50 \mathrm{ng}$ of DT. The efficiency of DC depletion in corneas was monitored by whole mound immunostaining before and 24 hours after wounding (hpw).

\section{Corneal Epithelial Debridement Wound}

Mice were anesthetized by i.p. injection of ketaminexylazine, and the central corneal epithelium was demarcated with a 2-mm trephine and then removed using a blade under a dissecting microscope. Care was taken to minimize injury to the epithelial basement membrane and stroma. While under anesthesia, ocular surfaces were protected from drying by topical administration of bacitracin ophthalmic ointment immediately after injury.

Assessment of wound closure was performed by fluorescein staining $(0.1 \%$ sterile fluorescein solution in PBS) and followed by rinsing of the ocular surface with PBS and photographing with a digital camera. The remaining denuded area was quantitated using Photoshop (Adobe System Inc., San Jose, CA). The healing rate was calculated as follows: (original wound area-current wound area)/original wound area (in percentage).

\section{Immunostaining of Whole Mount Corneal Tissue}

Mice were euthanized, and the entire cornea plus the limbus was excised under the operating microscope. Excised corneas were fixed in 4\% paraformaldehyde and stored at $4^{\circ} \mathrm{C}$ until further processing. Before staining, radial incisions were made to produce six pie-shaped wedges. Corneas were washed in PBS, incubated in 20 $\mathrm{mmol} / \mathrm{L}$ prewarmed EDTA for 30 minutes at $37^{\circ} \mathrm{C}$, and incubated with a $0.2 \%$ solution of Triton X-100 in PBS plus $1 \%$ bovine serum albumin (BSA) for 20 minutes at room temperature. After blocking, the corneas were incubated overnight at $4^{\circ} \mathrm{C}$ with $100 \mu \mathrm{L}$ of mouse CD11C antibody (BD Pharmingen, San Diego, CA) diluted in PBS with 1\% BSA. The tissues were then washed five times in PBS. Corneas were then incubated with $100 \mu \mathrm{L}$ Cy3-conjugated antibody diluted in PBS with 1\% BSA for 1 hour at room temperature. This was followed by five washes in PBS. Stained tissue whole mounts were placed in Vectashield mounting medium (Vector Lab, Burlingame, CA) onto glass slides and coverslipped. Corneal whole mounts were examined using confocal microscopy (TCSSP2; Leica, Wetzlar, Germany) for EGFP ${ }^{+}$cells or immunopositive cells in the corneal epithelium. 


\section{IHC and TUNEL Assay}

Mouse eyes were enucleated and embedded in TissueTek (Miles Inc., Elkhart, IN) optimal cutting temperature $(\mathrm{OCT})$ compound and frozen in liquid nitrogen. Sections (8- $\mu \mathrm{m}$ thick) were cut and mounted to polylysine-coated glass slides. After a 10-minute fixation in acetone, slides were blocked with $10 \mathrm{mmol} / \mathrm{L}$ sodium phosphate buffer containing 2\% BSA for 1 hour at room temperature. Sections were then incubated with mouse primary antibody [NIMP-R14 (anti-neutrophil antibody), 1:100; thymic stromal lymphopoietin (TSLP), 1:400; CXCL10, $5 \mu \mathrm{g} / \mathrm{mL}$; or p-AKT,1:100]. This was followed by a secondary antibody, fluorescein isothiocyanate, or Cy3-conjugated goat anti-rat or anti-rabbit IgG (Jackson ImmunoResearch Laboratories, West Grove, PA, 1:100), and slides were mounted with Vectorshield mounting medium containing DAPI mounting media. Controls were similarly treated, but the primary antibody was replaced with rat or rabbit lgG. To detect apoptotic cells, mouse corneal cryostat sections were fixed in $2 \%$ paraformaldehyde and stained with an ApopTag plus fluorescein in situ apoptosis detection kit (TUNEL staining; Chemicon, Temecula, CA). The stained slides were mounted with Vectorshield mounting DAPI medium for nuclear staining and examined under an Olympus BX5OF fluorescence microscope (Olympus, Tokyo, Japan) with an ApoTome digital camera.

\section{RNA Extraction and Real-Time PCR}

For RNA isolation, epithelial cells were scraped off the cornea and frozen in liquid nitrogen immediately. RNA was extracted from the collected epithelial cells using an RNeasy Mini Kit (Qiagen, Valencia, CA), according to the manufacturer's instructions. cDNA was generated with an oligo(dT) primer (Invitrogen, Carlsbad, CA), followed by analysis using real-time PCR with the Power SYBR Green PCR Master Mix (AB Applied Biosystems, Carlsbad, CA), based on expression of $\beta$-actin. The primer pairs used are in Table 1.

\section{Statistical Analyses}

Data were presented as mean $\pm \mathrm{SD}$. Statistical differences among three or more groups were identified using one-way analysis of variance. Differences were considered statistically significant at $P<0.05$.

\section{Results \\ DT-Mediated Local DC Depletion in the Cornea of CD11C-DTR Mice}

DCs were sparsely distributed centrally and were denser in the peripheral cornea, with fine processes inserting between epithelial cells and extending in the anteroposterior direction. ${ }^{11}$ By using confocal fluorescence microscopy, we confirmed the distribution pattern of DCs in CD11c-DTR mice: relatively high density near the limbus, less dense in the middle region, and sparse in the central corneas (Figure 1). The CD11c-stained cells were also expressing EGFP driven by a CD11c promoter in the transgenic mice. Virtually all CD11c-positive cells show dendritic morphological features in unstimulated corneas.

To study DC function, systematic administration of DT was generally used to deplete all DCs from CD11c-DTR mice. ${ }^{14,32,33}$ In this study, we determined if DCs can also be depleted locally by comparing the efficiency of systematic (i.p. injection) and local (s.c. injection) administrations of DT in the cornea. Figure 2 shows that, although i.p. injection of DT depletes DCs in both the cornea and the spleen, s.c. injection of DT effectively ablates DCs in the cornea and limbal region but exhibits no effects in the spleen. DCs in the cornea are morphologically different from those in the spleen. Examinations of CD11c-DTR murine corneas by slit lamp and ConfoScan 4 (NIDEK Technologies Srl, Padova, Italy), a digital confocal scanning microscope, showed no detectable abnormality in the ocular surface and in the morphological features between PBSand DT-injected corneas (data not shown). Thus, s.c. injection of DT was chosen for the study because the effects are limited to the ocular surface.

\section{Impaired Epithelial Wound Healing in DC-Depleted Corneas}

After having shown effective local depletion of DCs in the cornea, we next investigated their effects on corneal epithelial wound healing. An epithelial wound was generated by epithelial debridement at the center of the cornea using a 2-mm trephine to mark the wound size, leaving the basement membrane intact. Fluorescein staining over the cornea indicated the area of the cornea denuded of epithelium (Figure 3A). The wounds were approximately 95\% covered in the PBS-injected corneas, whereas 57\% still remain in DT-injected corneas (Figure 3B). To exclude the effect of S.c. injected DT on wound closure, we also used WT B6 mice injected with DT; healing rates of

Table 1. Primers Used for Real-Time PCR

\begin{tabular}{|c|c|c|}
\hline Gene & Forward primer & Reverse primer \\
\hline$\beta$-Actin & $5^{\prime}$ - GACGGCCAGGTCATCACTATTG-3' & $5^{\prime}-\mathrm{AGGAAGGCTGGAAAAGAGCC}-3^{\prime}$ \\
\hline TSLP & $5^{\prime}$-AGGCTACCCTGAAACTGAG- $3^{\prime}$ & $5^{\prime}$-GGAGATTGCATGAAGGAATACC $-3^{\prime}$ \\
\hline CXCL10 & $5^{\prime}-\mathrm{CCATCAGCACCATGAACCCAAGT-3^{ \prime }}$ & $5^{\prime}-\mathrm{CACTCCAGTTAAGGAGC-3^{ \prime }}$ \\
\hline SIL-1RN & $5^{\prime}$-CCTACAGTCACCTAATCTCTCT-3' & 5'-ATTGGTCTTCCTGGAAGTA-3' \\
\hline$I L-1 \beta$ & $5^{\prime}$-GGAGAAGCTGTGGACGCTA-3' & $5^{\prime}$-GCTGATGTACCAGTTGGGGA-3' \\
\hline
\end{tabular}




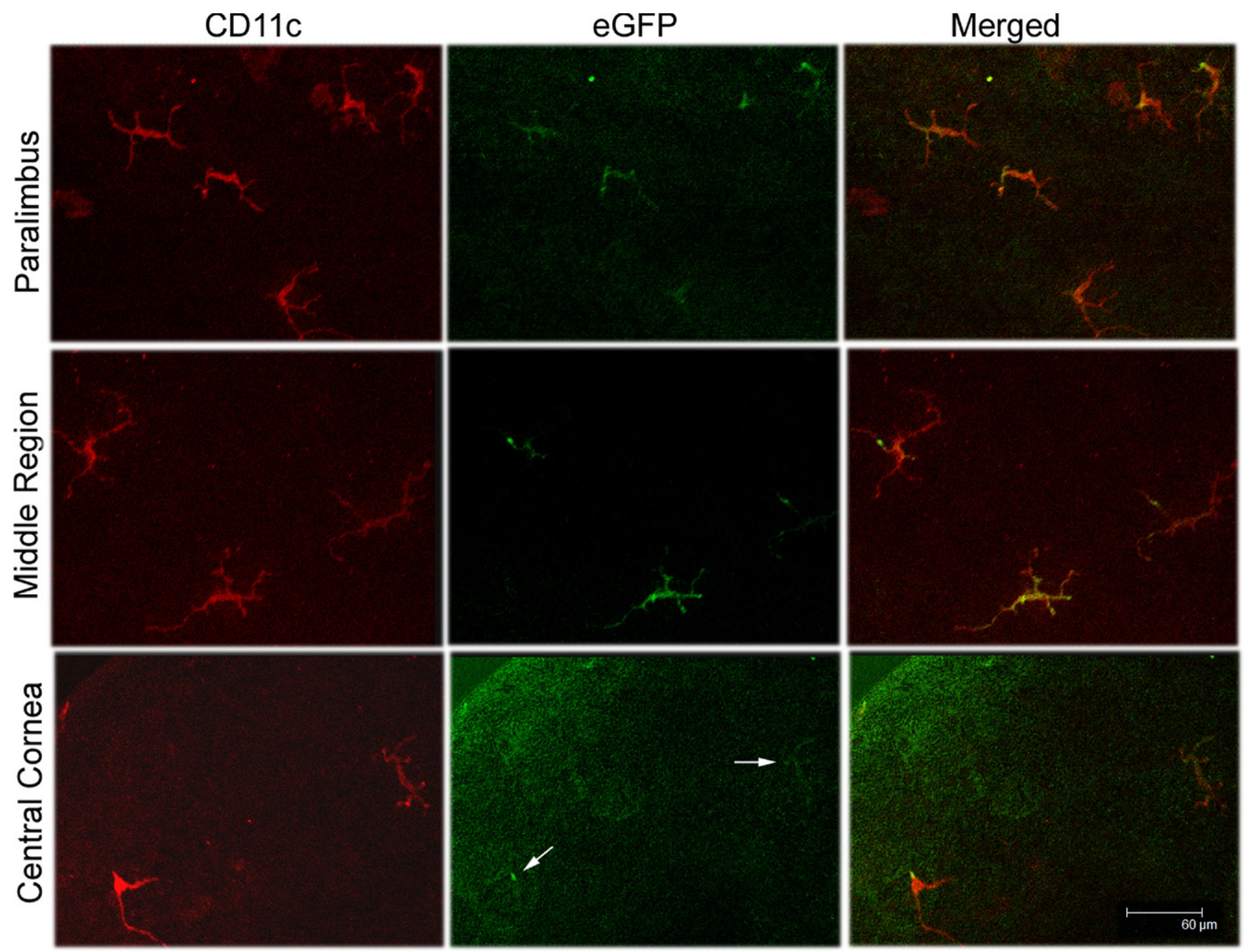

Figure 1. Whole mount confocal microscopy showing the distribution of intraepithelial DCs at different regions of CD11c-DTR mouse cornea. The corneas were stained for CD11c (red) and examined for EGFP (green). Arrows indicate EGFP (bottom center panel). DC density decreases from the peripheral to the central cornea. The figures are representative of images from six corneas $(n=3$ mice)

this control group were similar to those of CD11c-DTR mice injected with PBS (Figure 3), suggesting that s.c. DT has no or minimal effects on corneal epithelial wound healing. All epithelial wounds were healed by $26 \mathrm{hpw}$ in the transgenic mice receiving a PBS injection and in the WT B6 mice receiving a DT injection. However, in the DC-depleted transgenic mice receiving DT, wounds were not covered until 30 to $32 \mathrm{hpw}$. We concluded that DC deficiency in the cornea impairs epithelial wound closure in vivo.

\section{DCs Migrate along the Healing Epithelium in Injured B6 Mouse Corneas}

Because epithelial debridement should remove embedded DCs as well, we assessed DC migration and repopulation in the healing corneas. We used EGFP and CD11c staining to trace DCs that entered the healing corneas (Figure 4). In PBS-injected corneas at a low magnification, DCs were found from the leading edge to the limbal region. At high magnification, CD11c antibody staining, EGFP, and their merged images show dendritic morpho- logical features. Because this region is close to the center of the cornea, the density of DCs shown herein, compared with those shown in Figure 1, was higher in the healing corneas than that of the normal controls. In DTinjected corneas, CD11c-positive cells were not detected, suggesting that s.c. DT depletes residential DCs and also abates potentially recruited or infiltrating DCs in the cornea. We conclude that DCs and epithelial cells form a migration sheet to cover the wound and that DCs are an integrated part of the epithelial wound-healing apparatus in the cornea.

\section{DCs Affect the Gene Expression of the Migrating Epithelial Sheet}

After having shown the presence of DCs in migratory epithelia, we next investigated the effects of DC depletion on the expression of cytokines and cytokine antagonists in healing corneal epithelia using real-time PCR (Figure 5). The RNA for PCR is prepared from scraped epithelial sheets that contain mostly epithelial cells and embedded DCs, assuming there is no mRNA in the nerve ends. In 
Subconjectival I
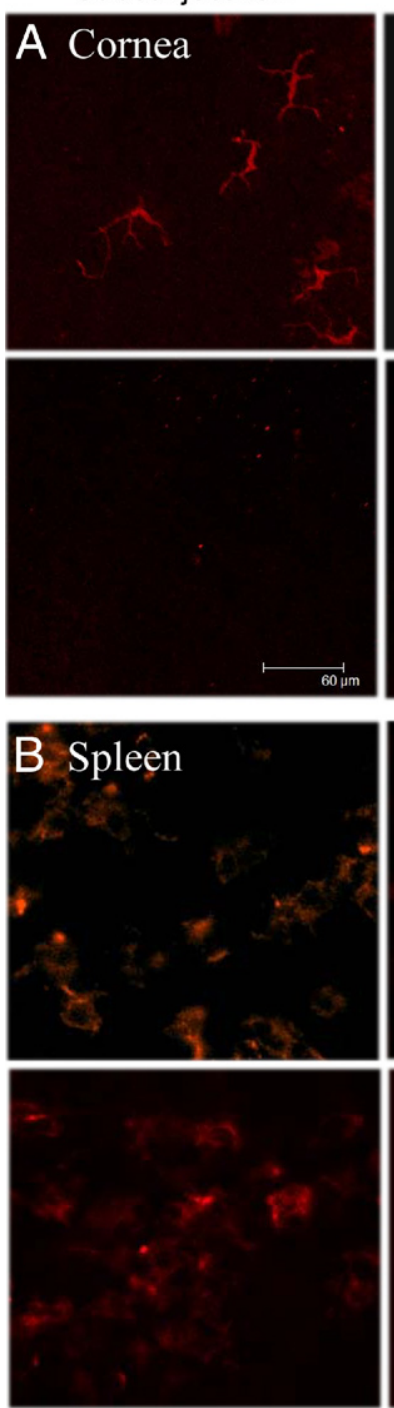

Figure 2. Confocal microscopy of corneal whole mount and spleen sections showing depletion of DCs in CD11c-DTR mice. CD11c-DTR mice were injected with $100 \mathrm{ng}$ of DT in $100 \mu \mathrm{L}$ of PBS i.p. or $5 \mathrm{ng}$ of DT in $5 \mu \mathrm{L}$ of PBS s.c., with PBS alone as the control. The corneas were excised 24 hours after DT injections, stained with CD11c antibody, and examined with confocal microscopy for the cornea (A, whole mount) or epifluorescence microscopy for the spleen (B, cryostat sections). The figure is a representative of four corneas $(n=2$ mice) per condition.

each group, naïve untreated mice were used as the base of expression. In CD11c-DTR mice, all four genes showed a pattern of differential expression in the DCdepleted migratory corneal epithelial sheet (Figure 5B). Among the genes tested, CXCL10 (IP-10) exhibited the most dramatic increase after injury, and this up-regulation is attenuated by DC depletion. TSLP is an epithelialexpressed cytokine that recruits and activates immune cells, including DCs. ${ }^{34,35}$ Its expression was increased in migratory epithelia, and the lack of DCs reduced woundinduced TSLP expression to a level similar to the controls. $\mathrm{IL}-1 \beta$ and its receptor antagonist, sIL-1Ra, are paired in many tissues to control inflammation. ${ }^{36}$ Their expressions in injured corneas were up-regulated, and DC depletion resulted in 1.4- and 3.2-fold decreases for $\mathrm{IL}-1 \beta$ and
slL-1Ra, respectively. Intriguingly, the highest level of sIL-1Ra was found in uninjured DC-depleted corneal epithelia, in which no significant increase in IL- $1 \beta$ was observed. To ensure that the altered gene expression in CD11c-DTR mice was not due to s.c. DT, WT B6 mice were used as the control (Figure 5A). Wounding resulted in a similar pattern of gene expression in PBS-injected corneas, and s.c. DT had little effects on wound-induced expression of CXCL10,IL-1 $\beta, T S L P$, and $S / L-1 R a$ in the corneas of B6 mice, suggesting minimal effects of s.c. DT on normal corneal epithelial cells. Thus, we conclude that the reduced expression of these four genes in corneal epithelial cells is due to the lack of DCs.

The altered expressions of TSLP and CXCL10 in DCdepleted corneas were also assessed by immunohistochemistry (IHC; Figure 6). In uninjured corneas with or without DT injections, there was negative staining for both TSLP and CXCL10, suggesting low or no expression of the genes. In injured corneas, TSLP was found in the cells of the leading edge, whereas CXCL10 was found near the leading edge, starting approximately 10 cells away from the first cell at the tip of the leading edge in PBSinjected corneas. On the other hand, only faint staining was observed for both cytokines in DT-injected corneas. These two cytokines were generally not detected in the stroma of migratory epithelia. We concluded that healing epithelia express cytokines that recruit and/or activate DCs and that the presence of DCs is required for their wound-induced cytokine expression in the cornea.

\section{Lack of DCs Results in an Increase in Cell Death in the Cornea}

Epithelial wound closure requires cell sliding (ie, reverse differentiation of wing cells to become basal cell like); cell migration driven by the basal cells, especially those at the leading edge; and proliferation distant from the leading edge to repopulate the lost cells. ${ }^{21,37}$ In previous studies, ${ }^{38,39}$ delayed epithelial wound closure in diabetic cornea was related to the increase in epithelial apoptosis or TUNEL-positive cells. Therefore, we performed TUNEL staining in wounded corneas. We first assessed the stromal cell apoptosis at $3 \mathrm{hpw}$, when epithelial migration had not started (Figure 7). Previous studies ${ }^{40,41}$ have shown that epithelial scraping results in rapid apoptosis of stromal fibroblasts. As expected, there were many TUNEL-positive cells in the stroma of epithelium-denuded corneas injected with either PBS or DT at $3 \mathrm{hpw}$. In healing corneas, there were no TUNEL-positive cells in the PBS-injected corneas. On the other hand, at the migratory phase (24 hpw), there were several TUNEL-positive cells in migratory epithelia and significantly more in the stroma. We also performed TUNEL staining of the corneas injected with PBS or DT without wounding. At 48 hours after injection (identical to $24 \mathrm{hpw}$ ), no dying cells were seen in the stroma. However, many epithelial cells were seen at the apical and subapical layers of the epithelium; these cells had a flattened shape, characteristic of differentiated apical corneal epithelial cells, were TUNEL positive, and had a 
A

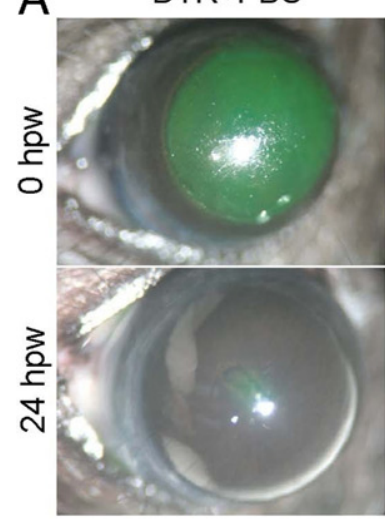

$\mathrm{DTR}+\mathrm{DT}$

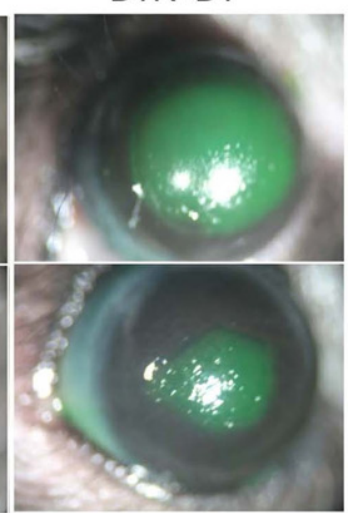

WT+DT

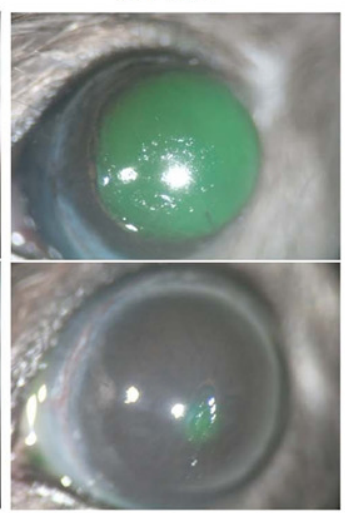

B

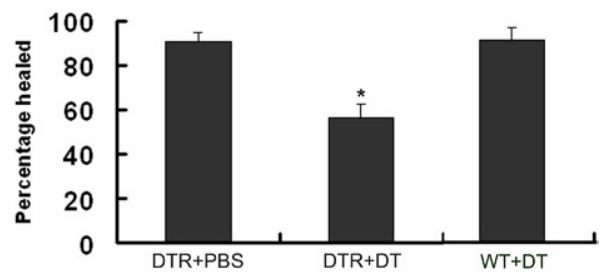

Figure 3. Impaired corneal epithelial wound closure in DC-depleted mice. Corneal wounds were made by scraping the epithelia of CD11c-DTR mice s.c. injected with PBS (DTR+PBS) or DT (DTR+DT) or WT mice s.c. injected with DT (WT+DT). Wounded corneas were stained with $0.25 \%$ fluorescein sodium immediately after wounding $(0 \mathrm{hpw})$ or at $24 \mathrm{hpw}$. A: Fluorescein staining of the original corneal epithelial wounds or at $24 \mathrm{hpw}$. The figure is representative of six corneas per condition from three independent experiments. B: The healing rate was expressed as the mean \pm SD from five eyes each. ${ }^{*} P<0.05$.

pattern similar to that observed in diabetic human and rat (both normal and healing) corneas. ${ }^{38,39}$

\section{Depletion Disturbs Epithelial Phosphatidylinositol 3-Kinase-AKT Signaling in the Cornea}

We previously showed that epidermal growth factor receptor (EGFR)-phosphatidylinositol 3-kinase-AKT plays a pivotal role in mediating epithelial survival and wound healing. ${ }^{20,38,39}$ We next investigated whether its signaling is altered by DC depletion using $\mathrm{IHC}$ with anti-phosphorylated-AKT antibodies (Figure 8). Although we were unable to detect phospho-AKT in uninjured corneas, cells at the leading edge of migratory epithelia were strongly stained with phospho-AKT antibody in the PBS-injected corneas, whereas there was no phospho-AKT presence in the DT-injected corneas. Thus, DCs influence the epithelial cell response in terms of signal transduction in response to wounding in the cornea.

\section{Lack of DCs Results in a Decrease in Neutrophil Infiltration in the Stroma}

Epithelial injury causes the influx of PMNs into the corneal stroma, and PMN infiltration has been important for proper wound healing. ${ }^{24}$ In TUNEL-staining micrographs (Figure 7), we also noticed numerous TUNEL-positive cells in the stroma and suspected that some of the dying
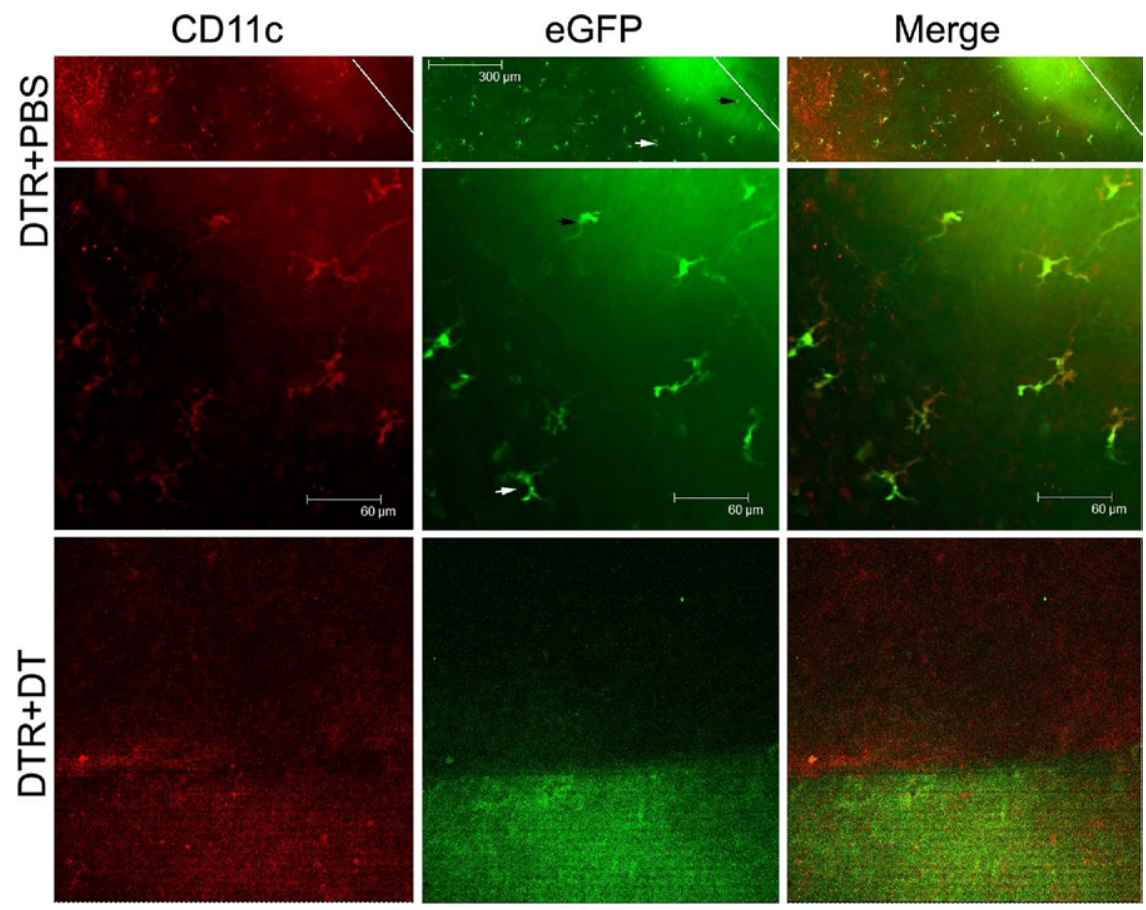

Figure 4. Whole mount confocal microscopy showing the distribution of DCs in healing corneal epithelia. Whole corneas of CD11c-DTR mice collected $24 \mathrm{hpw}$ were stained with CD11c antibody (Cy3, red) and examined by confocal microscopy for EGFP. Cy3 and EGFP images were merged. The leading edge of migrating epithelium is marked with a white line in lowmagnification images (top panel). The middle and bottom panels are under high magnification. Arrowheads mark the same cell at different magnifications. The figures are representative of three corneas per condition from two independent experiments. No CD11c-positive cells can be identified in the DT-injected cornea. 

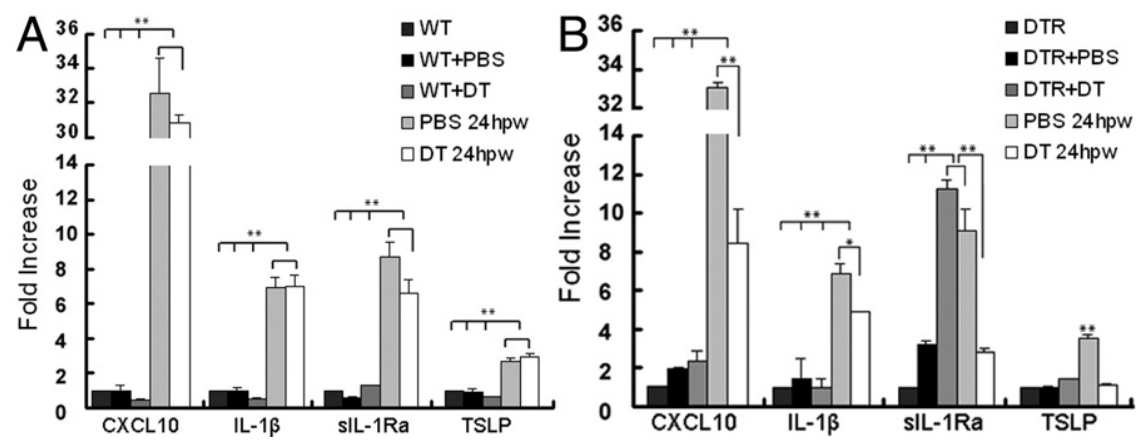

Figure 5. $\mathbf{A}$ and $\mathbf{B}$ : Real-time PCR showing differential expression of CXCL10, TSLP, IL- $1 \beta$, and sIL-Ra in DT- and PBS-injected corneas of CD11c-DTR (B), but not evident in WT B6 (A), mice. Corneal epithelial cells were scraped off of the following corneas: $i$ unmanipulated (DTR or WT), ii) s.c. injected with PBS (DTR+PBS or WT+PBS) or DT (DTR+DT or WT+DT) 24 hours after injections without wounding, or iii) s.c. injected with PBS or DT and then wounded for $24 \mathrm{hpw}$ (PBS $24 \mathrm{hpw}$ and DT $24 \mathrm{hpw}$, both $\mathbf{A}$ and $\mathbf{B}$ ) and processed for real-time PCR analysis, as described in Materials and Methods. Each sample was normalized with actin as the internal control, and the results were expressed as fold increase with epithelial cells from unmanipulated CD11c-DTR or WT mouse corneas as one. The results are the representative of two experiments, each with three samples derived from three mice $(n=2$ corneas pooled). ${ }^{*} P \leq 0.05$.

cells might be PMNs. Thus, we next investigate if PMN infiltration is also affected by DC depletion. As shown in Figure 9, there were no NIMP-R14 (a PMN cell marker) positive cells in normal uninjured corneas (without wounding), regardless of PBS or DT injection. In the corneas $24 \mathrm{hpw}$, several PMNs can be seen in the front and around the leading edge in both DT- and PBS-injected corneas, although fewer can be seen in DT-injected corneas. In the stroma at the anterior side of PBS-injected corneas, many NMP-R14-positive cells were found, whereas many fewer were found in DT-injected corneas. We conclude that DCs play a role in recruiting PMNs to the injured corneas.

\section{Discussion}

In this study, we investigated the role of DCs in corneal epithelial wound healing. We showed that DCs, which are normally found in the healthy cornea and are removed by epithelial scraping, migrate, along with healing the corneal epithelial sheet. We demonstrated that intraepithelial DCs can be depleted locally and that this depletion results in the significant delay of re-epithelialization of corneal wounds in CD11c-DTR mice. We also found that depletion of DCs significantly attenuated the wound-induced expression of CXCL10, TSLP, IL-1 $\beta$, and IL-1Ra and the cellular localization of TSLP and CXCL10 in healing the corneal epithelia. Moreover, there are significantly more apoptotic cells and a lower immunoreactivity in phospho-AKT for DT-treated corneas compared with the controls. Finally, depletion of DCs also resulted in a decrease in PMN infiltration into the healing cornea. Thus, although DCs are known for specializing in antigen presentation, our study identifies an additional physiological function of these cells in close interaction with surrounding epithelial cells and in participating tissue repair at the ocular surface.
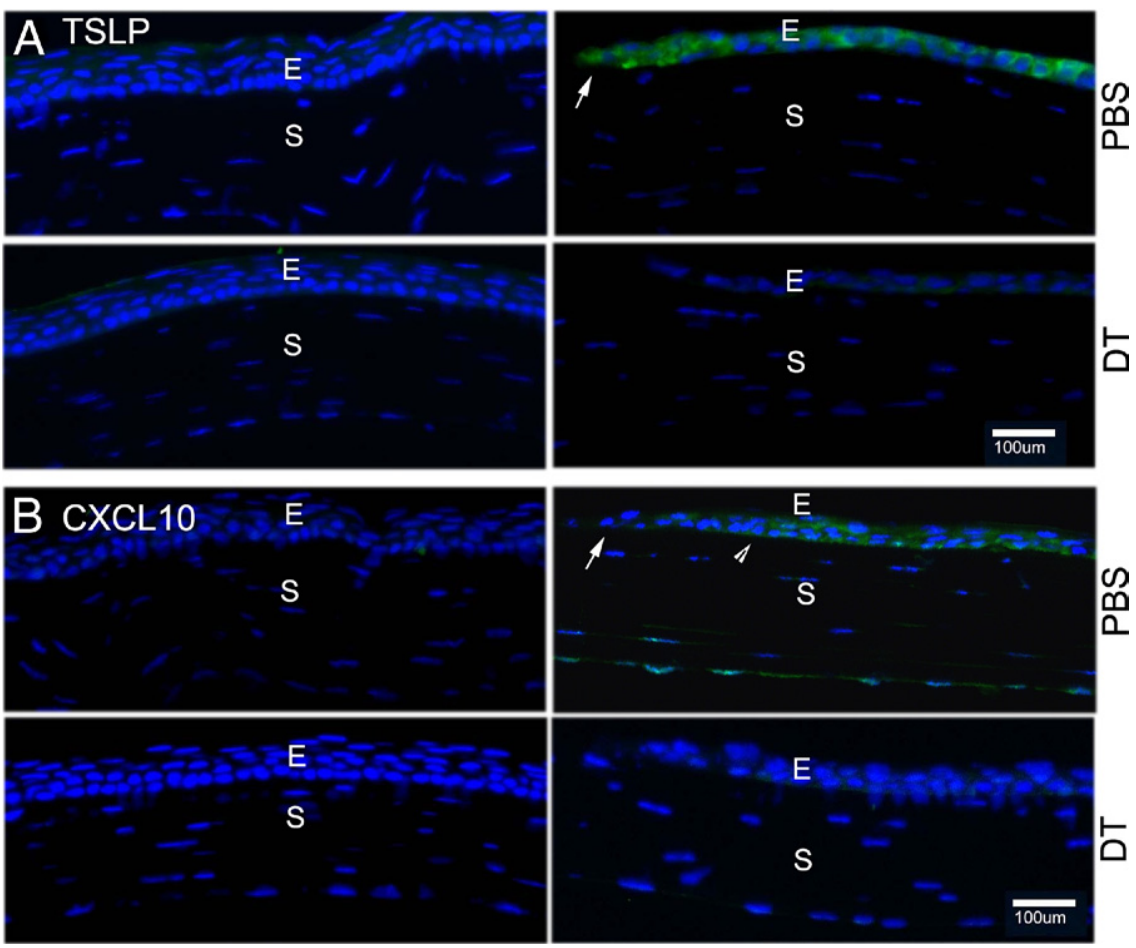

Figure 6. IHC of TSLP and CXCL10 distributions in DT-depleted corneas. Cryostat sections of DTor PBS-injected mouse corneas were immunostained with antibodies against TSLP (A, green) or CXCL10 (B, green) in the cornea $24 \mathrm{hpw}$ with DAPI in mounting media to illustrate nuclei (blue). The leading edge (arrowhead) and starting point (arrows) of elevated CXCL10 expression are shown. E, epithelium; S, stroma. The figure is representatives of four corneas per condition from two independent experiments. 


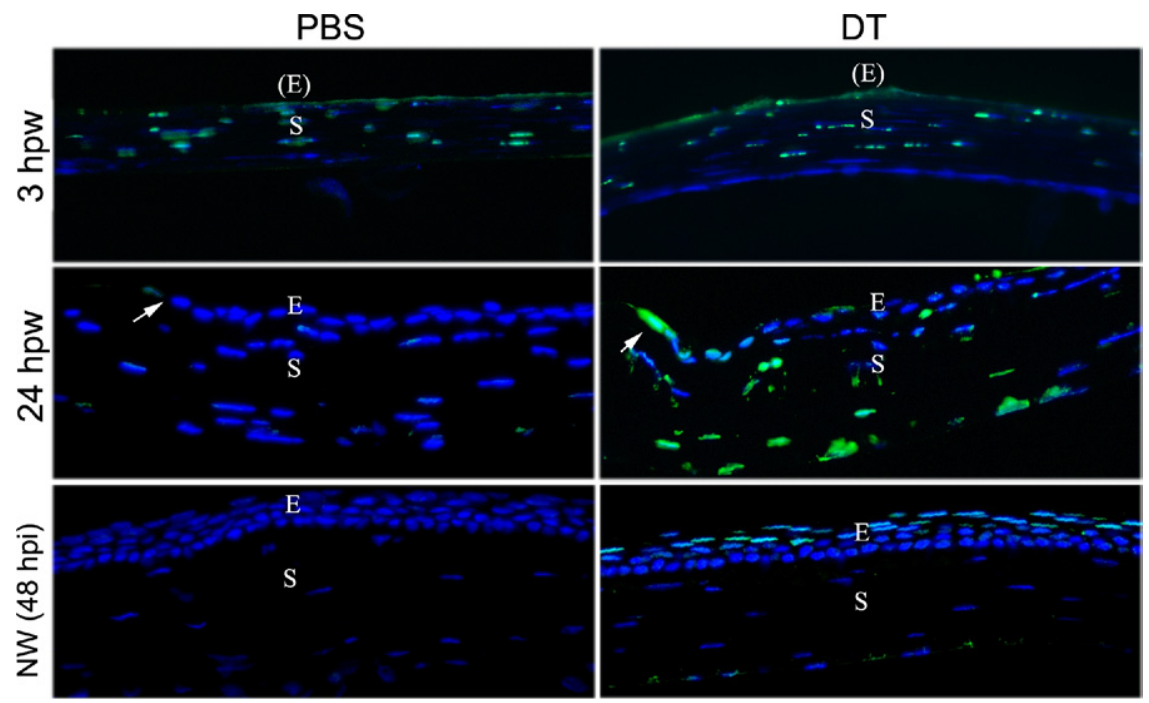

Figure 7. Increased cellular apoptosis in DCdepleted corneas assessed by TUNEL staining. CD11c-DTR mice were injected with PBS or DT and then subjected to epithelial debridement at 24 hours after injection. The corneas were collected at 3 and $24 \mathrm{hpw}$ or without wounding for 48 hours [NW (48 hpi)] and cryostat sectioned The sections were processed by TUNEL staining. Photographs show merged images of TUNEL and nuclear staining of DAPI. Arrows indicate the wound edge. The figure is a representative of three corneas per condition from two independent experiments. E, epithelium; (E), epithelium denuded; S, stroma.

CD11C-driven DTR-EGFP mice of different genetic backgrounds have been commonly used to deplete DCs. ${ }^{32}$ Although most studies use system administration of DT, which may cause mortality in DTR mice, ${ }^{42}$ the ocular surface is unique in that a local depletion, through s.c. injection of DT, is possible. In addition to being local, s.c. injection has another advantage because it is a routinely used procedure in the ophthalmologist's office, which allows reliable delivery of the desired amount to the ocular surface. Indeed, we showed that, although DT, through i.p. injection, resulted in the disappearance of DCs from both the spleen and the cornea, DT via s.c. injection ablated DCs in the cornea only. We demonstrate that depletion of DCs locally in the cornea significantly affected the epithelial response to wounding, resulting in much delayed re-epithelialization of CD11c-DTR mice compared with PBS-injected mice or DT-injected WT B6 mice. Thus, we conclude that DCs play a role in mediating corneal epithelial wound healing.

In normal corneas, epithelial DCs are sparsely distributed centrally and are denser in the periphery (Figure 1). ${ }^{11}$ These cells generally do not migrate laterally in unstimulated corneas or in corneas stimulated in the central region with silver nitrate burn injury, Escherichia coli lipopolysaccharide, or microspheres, all considered point injuries. ${ }^{11,13}$ Strik- ingly, we found that the DCs migrated toward the wound center, along with epithelial sheets, and that the density of DCs near the central cornea in healing corneas is much higher than that in normal corneas. These observations raise several interesting questions regarding DCepithelium interaction and function in epithelial homeostasis and wound healing. First, what are the sources of DCs in the healing cornea? The stroma, which also contains DCs, is an unlikely source because early studies $^{43,44}$ revealed that epithelial DCs migrate from the peripheral cornea but not from the stroma. In our wound model, the epithelium of the peripheral cornea, which has a relatively high density of DCs, remains and is a likely source for DCs migrating into the wound bed, although circulating DCs, through limbal vessels infiltrating into the injured cornea, cannot be excluded. Second, what are the mediators of DC recruitment and/or activation in healing corneas? Recent studies ${ }^{1,45,46}$ in different tissues revealed that epithelial cells are an important tissue sentinel that recognize danger signals and can initiate a rapid response to injury and other insults, including the release of cytokines and growth factors. These epitheliagenerated mediators may work in an autocrine or paracrine fashion to activate cells around injury sites, epithelial cells, and DCs in our model. To this end, we found that

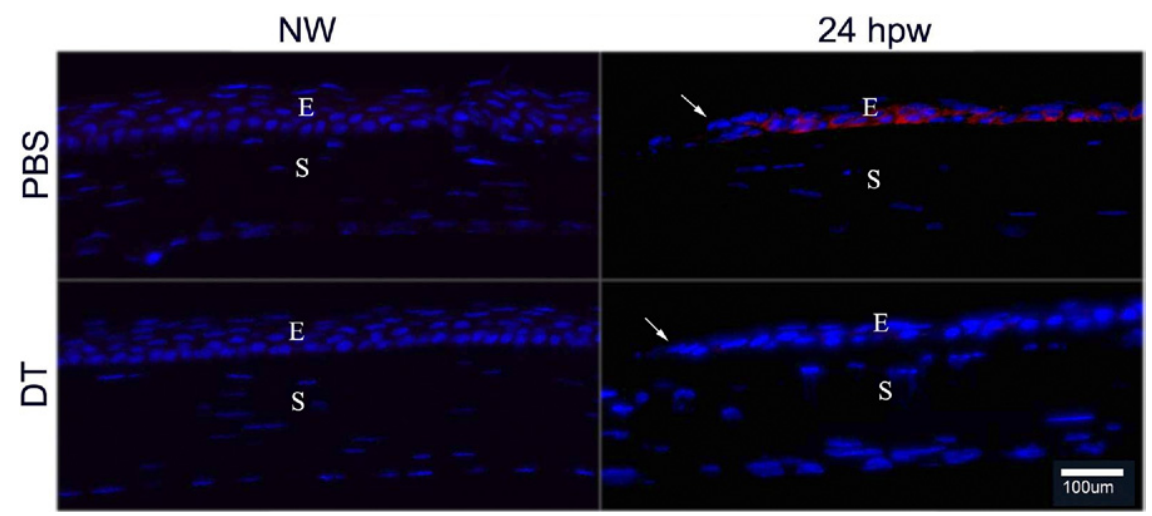

Figure 8. Phospho-AKT staining in the healing corneal epithelium of DT-depleted mouse corneas. Cryostat sections of PBS- or DT-injected mouse corneas, with wounding $(24 \mathrm{hpw})$ or without wounding (NW), were immunostained with antibody against phospho-AKT (Ser473). Photographs show merged images of immunofluorescence of phospho-AKT (red) and nuclear staining of DAPI (blue). Arrows mark the leading edge of migration corneal epithelial cells The figures are representatives of four corneas per condition from two independent experiments. E, epithelium; S, stroma. 

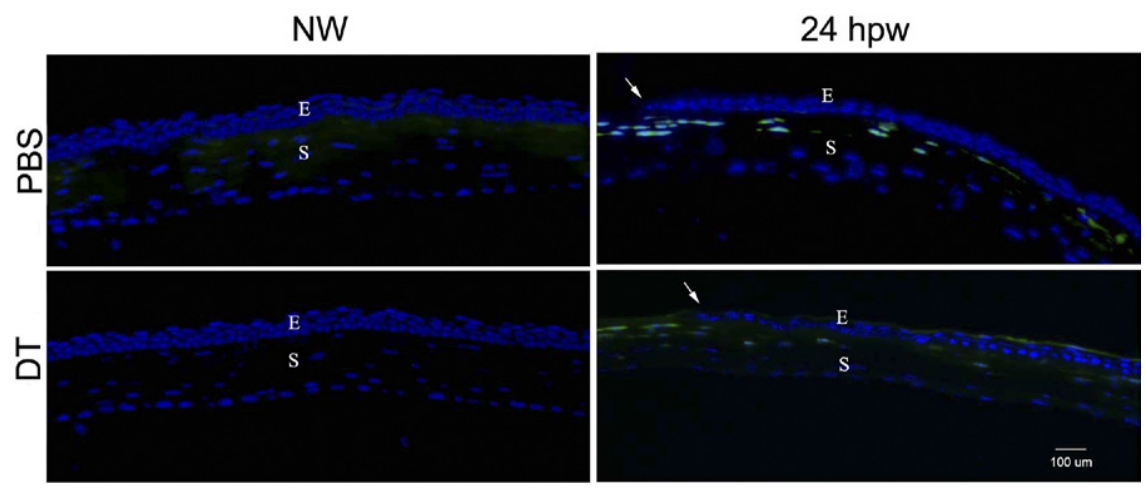

Figure 9. PMN recruitment in the healing corneal epithelium of DT-depleted mouse corneas. Cryostat sections of PBS- or DT-injected mouse corneas were immunostained with NIMP-R14 to detect PMNs. Photographs show merged images of immunofluorescence of phospho-AKT (red) and nuclear staining of DAPI (blue). Arrows mark the leading edge of migration corneal epithelial cells. The figure is representative of three corneas per condition from two independent experiments. E, epithelium; S, stroma.

TSLP and CXCL10 are highly expressed in migrating epithelia during wound healing.

TSLP is an epithelium-derived cytokine that strongly activates DCs through interaction with the TSLP receptor expressed by the DCs. ${ }^{47,48}$ CXCL10 is chemotactic for natural killer cells, activated T cells, and DCs. ${ }^{49}$ Little information exists on the potential role of CXCL10 and its receptor, C-X-C chemokine receptor 3 , or TSLP in wound healing. ${ }^{50}$ Therefore, based on the data presented herein, we propose that migratory epithelial cells, particularly those at the leading edge, activate DCs and direct their migration/infiltration into the wound bed through the release of TSLP and/or CXCL10. Also, TSLP is expressed in the front of CXCL10, suggesting a role of TSLP as DCs' chemoattractant, an unidentified function for the protein, whereas CXCL10 may serve as a cytokine and/or chemokine for the intraepithelial DCs. In addition to these two DC-targeting cytokines, we also found elevated expressions of IL-1 $\beta$ and its antagonist SIL-1Ra. Because IL-1 $\beta$ is a highly active and pleiotropic pro-inflammatory cytokine, its activity is tightly controlled by several naturally occurring inhibitors, such as IL-1Ra. ${ }^{36}$ An imbalance between IL-1 and IL-1Ra has caused a variety of human diseases, primarily of epithelial and endothelial cell origin. ${ }^{51}$ Although wound-induced expressions of both $\mathrm{IL}-1 \beta$ and IL-1Ra are attenuated by DC depletion, the decrease in sIL-1Ra is much more profound than that in IL-1 $\beta$ (1.4- and 3.2-fold decreased, respectively), suggesting an imbalance favoring IL-1 $\beta$ or inflammation in DT-depleted healing corneas. Although the expression of TSLP and CXCL10, assessed by real-time PCR, was confirmed by $\mathrm{IHC}$, the expression of $\mathrm{IL}-1 \beta$ and $\mathrm{IL}-1 \mathrm{Ra}$ was not confirmed by $\mathrm{IHC}$. Because one reference gene (ie, actin) was used, ${ }^{52}$ further studies to confirm the expression of genes at the protein levels and to assess how this imbalance might influence corneal tissue repair and inflammation are warranted. Interestingly, in DC-depleted corneas, but not in PBS-injected CD11c-DTR mice or DT-injected WT B6 mice, the highly elevated expressions of TSLP and CXCL10 were greatly attenuated, suggesting a strong positive feedback loop between epithelial and intraepithelial DCs. To our knowledge, this is the first report of such a system existing in mucosal epithelial surfaces. An understanding of the feedback mechanisms may open an avenue for studying mucosal surface homeostasis and tissue repair and might lead to new tar- gets for treating wound healing-related diseases, such as impaired diabetic wound healing and cancer metastases.

The key role of intraepithelial DCs in wound response is further illustrated by the observation that DT depletion results in cell death in migrating epithelia, whereas none are found in the control corneas. To our knowledge, this is the first study identifying a role for intraepithelial DCs in promoting epithelial survival in a mucosal tissue under normal and pathogenic (injury) conditions. Apoptosis of migrating epithelial cells is a surprise because wounding activates EGFR signaling, including the EGFR-phosphatidylinositol 3-kinase-AKT pathway, which prevents epithelial apoptosis. ${ }^{20,38,39}$ Indeed, we observed that an elevation of phospho-AKT in migrating epithelia and DT injection greatly reduced this elevation in healing corneal epithelia. Lack of phospho-AKT, an anti-apoptosis and prosurvival intracellular signaling effector, may contribute to the TUNEL-positive cells seen in healing corneal epithelia. ${ }^{53}$ Thus, intraepithelial DCs are the first immune cells that sense cell injury, either directly, through the recognition of danger signals [eg, extracellular ATP or high mobility group box chromosomal protein 1 (HMGB1) released by injured epithelial cells], or indirectly, from signals (ie, cytokines and chemokines) emanating from epithelia around the injury site. ${ }^{2,45,54}$ Moreover, numerous TUNEL-positive cells are found at the apical layers of healed and uninjured (data not shown) corneas lacking DCs. In normal corneas, there are no apoptotic cells because turnover of these cells depends on the sloughing off of superficial cells that are carried away by tears. ${ }^{55}$ Interestingly, the pattern of TUNEL-positive cells in DCdepleted corneas is similar to that observed in diabetic corneas of rats (wounded and normal) and humans. ${ }^{38,39}$ This leads us to speculate that DCs might also be a target of hyperglycemia and that their dysfunction after prolonged exposure to it may contribute to impaired wound healing in the diabetic skin and cornea. From the current study, it is not clear if DCs act on epithelial cells through cell-cell contact or through the release of cytokines (eg, IL-6) and growth factors (eg, EGFR ligands), both of which enhance epithelial wound healing. ${ }^{19,20,56}$ Taken together, our data suggest that activated intraepithelial DCs may secrete growth or survival factors and/or interact with epithelial cells through cell-cell contact to modulate migration, proliferation, and/or survival of epithelial 
cells. These epithelial cells, in turn, produce cytokines and chemokines, including TSLP and/or CXCL10, to further recruit and/or activate DCs in the wounding area. Altered cell signaling and cell survival in DC-depleted corneas may be contributing factors for delayed epithelial wound healing. These results provide strong evidence that DCs conspire or interplay with epithelial cells to form a functional unit for the homeostasis and wound healing of the cornea and other tissues.

In addition to directly interacting with epithelial cells, wound-activated DCs may indirectly contribute to the healing process by controlling the inflammatory response. A certain level of inflammation is required for efficient wound healing. ${ }^{57,58}$ Consistent with this notion, we observed that $\mathrm{DC}$ depletion results in decreases in the expression of pro-inflammatory cytokines, such as CXCL10, TSLP, and IL-1 $\beta$, and in the recruitment of PMNs in healing corneas. PMNs have entered the corneal stroma shortly after epithelial injury, and their presence appears to facilitate wound closure. ${ }^{24,59}$ Although the mechanism of the action of PMNs in epithelial wound healing is not clear, they could act as effector cells downstream of wound-activated DCs to influence epithelial wound healing. A decrease in infiltrated PMNs in DCimpaired corneas could be the result of reduced recruitment and/or increased apoptosis. In the present study, TUNEL staining revealed that, although there are no TUNEL-positive cells in the control PBS-injected corneas, numerous apoptotic cells are found in the stroma of healing corneas of DT-injected CD11c-DTR mice. These dying cells in the stroma are likely PMNs because the repopulating stromal fibroblasts always lag behind migrating epithelial sheets. ${ }^{60}$ Thus, apoptosis is likely a contributing factor to the lack of PMNs in DC-depleted healing corneas. Presently, it is not clear if DC depletion also causes defects in wound-induced PMN recruitment and/or emigration. Moreover, although this study is primarily focused on intraepithelial DCs, it is plausible that s.c. DT may also cause the depletion of stromal DCs that have recently been shown to be morphologically and functionally different from intraepithelial DCs. ${ }^{10}$ Nevertheless, our results clearly demonstrate an important regulatory role of DCs (intraepithelial and/or stromal) in controlling the function of PMNs in the cornea. Further study to define the roles of DC subtypes in corneal epithelial wound healing, including the use of Langerhans cellspecific depletion via s.c. administration of DT to Langerin DTR mice, ${ }^{10}$ is warranted.

In conclusion, our study revealed that corneal epithelial cells actively participate in the regulation of DC migration, infiltration, and activation in the cornea in response to wounding. Moreover, we identified a critical role of DCs in mediating corneal wound healing or reepithelialization. These findings provide a paradigm shift in understanding the function of DCs from the classic view of antigen-presenting cells in the recognition of infectious pathogens to partnering with epithelial cells for tissue repair and maintenance. Our findings in the cornea are likely applicable to other tissues, such as the skin and airway, and other pathological conditions, such as delayed wound healing in the diabetic skin and cornea.

\section{Acknowledgments}

We thank all of the members of the Yu and Mi laboratories for assistance and comments on the work and Jenny $\mathrm{Mi}$ for proofreading the manuscript.

\section{References}

1. Soloff AC, Barratt-Boyes SM: Enemy at the gates: dendritic cells and immunity to mucosal pathogens. Cell Res 2010, 20:872-885

2. Iwasaki A: Mucosal dendritic cells. Annu Rev Immunol 2007, 25:381418

3. Tournier JN, Mohamadzadeh M: Key roles of dendritic cells in lung infection and improving anthrax vaccines. Trends Mol Med 2010, 16:303-312

4. Tezuka $\mathrm{H}$, Ohteki T: Regulation of intestinal homeostasis by dendritic cells. Immunol Rev 2010, 234:247-258

5. Hamrah P, Dana MR: Corneal antigen-presenting cells. Chem Immunol Allergy 2007, 92:58-70

6. Lambrecht BN, Hammad H: The role of dendritic and epithelial cells as master regulators of allergic airway inflammation. Lancet 2010 , 376:835-843

7. Segawa K: Electron microscopic studies on the human corneal epithelium: dendritic cells. Arch Ophthalmol 1964, 72:650-659

8. Hamrah P, Liu Y, Zhang Q, Dana MR: The corneal stroma is endowed with a significant number of resident dendritic cells. Invest Ophthalmol Vis Sci 2003, 44:581-589

9. Hamrah P, Zhang Q, Liu Y, Dana MR: Novel characterization of MHC class II-negative population of resident corneal Langerhans cell-type dendritic cells. Invest Ophthalmol Vis Sci 2002, 43:639-646

10. Hattori T, Chauhan SK, Lee H, Ueno H, Dana R, Kaplan DH, Saban DR: Characterization of Langerin-expressing dendritic cell subsets in the normal cornea. Invest Ophthalmol Vis Sci 2011, 52:4598-4604

11. Lee EJ, Rosenbaum JT, Planck SR: Epifluorescence intravital microscopy of murine corneal dendritic cells. Invest Ophthalmol Vis Sci 2010, 51:2101-2108

12. Chinnery HR, Pearlman E, McMenamin PG: Cutting edge: membrane nanotubes in vivo: a feature of $\mathrm{MHC}$ class $\mathrm{II}+$ cells in the mouse cornea. J Immunol 2008, 180:5779-5783

13. Ward BR, Jester JV, Nishibu A, Vishwanath M, Shalhevet D, Kumamoto T, Petroll WM, Cavanagh HD, Takashima A: Local thermal injury elicits immediate dynamic behavioural responses by corneal Langerhans cells. Immunology 2007, 120:556-572

14. Mott KR, Ghiasi H: Role of dendritic cells in enhancement of herpes simplex virus type 1 latency and reactivation in vaccinated mice. Clin Vaccine Immunol 2008, 15:1859-1867

15. Hamrah $P$, Huq SO, Liu Y, Zhang Q, Dana MR: Corneal immunity is mediated by heterogeneous population of antigen-presenting cells. $\mathrm{J}$ Leukoc Biol 2003, 74:172-178

16. Swamy M, Jamora C, Havran W, Hayday A: Epithelial decision makers: in search of the "epimmunome." Nat Immunol 2010, 11:656665

17. Karrasch $T$, Jobin C: Wound healing responses at the gastrointestinal epithelium: a close look at novel regulatory factors and investigative approaches. Z Gastroenterol 2009, 47:1221-1229

18. Nishida T: Translational research in corneal epithelial wound healing Eye Contact Lens 2010, 36:300-304

19. Yu FS, Yin J, Xu K, Huang J: Growth factors and corneal epithelial wound healing. Brain Res Bull 2010, 81:229-235

20. Xu KP, Ding Y, Ling J, Dong Z, Yu FS: Wound-induced HB-EGF ectodomain shedding and EGFR activation in corneal epithelial cells. Invest Ophthalmol Vis Sci 2004, 45:813-820

21. Lu L, Reinach PS, Kao WW: Corneal epithelial wound healing. Exp Biol Med (Maywood) 2001, 226:653-664

22. Chikama T, Wakuta M, Liu Y, Nishida T: Deviated mechanism of wound healing in diabetic corneas. Cornea 2007, 26:S75-S81

23. Pflugfelder SC: Is autologous serum a tonic for the ailing corneal epithelium? Am J Ophthalmol 2006, 142:316-317

24. Petrescu MS, Larry CL, Bowden RA, Williams GW, Gagen D, Li Z, Smith CW, Burns AR: Neutrophil interactions with keratocytes during corneal epithelial wound healing: a role for CD18 integrins. Invest Ophthalmol Vis Sci 2007, 48:5023-5029 
25. Li Z, Burns AR, Rumbaut RE, Smith CW: $\gamma \delta$ T cells are necessary for platelet and neutrophil accumulation in limbal vessels and efficient epithelial repair after corneal abrasion. Am J Pathol 2007, 171:838845

26. Gronert K, Maheshwari N, Khan N, Hassan IR, Dunn M, Laniado Schwartzman M: A role for the mouse 12/15-lipoxygenase pathway in promoting epithelial wound healing and host defense. J Biol Chem 2005, 280:15267-15278

27. Chinnery HR, Carlson EC, Sun Y, Lin M, Burnett SH, Perez VL, McMenamin PG, Pearlman E: Bone marrow chimeras and C-fms conditional ablation (Mafia) mice reveal an essential role for resident myeloid cells in lipopolysaccharide/TLR4-induced corneal inflammation. J Immunol 2009, 182:2738-2744

28. Pearlman E, Johnson A, Adhikary G, Sun Y, Chinnery HR, Fox T, Kester M, McMenamin PG: Toll-like receptors at the ocular surface. Ocul Surf 2008, 6:108-116

29. Chinnery HR, Ruitenberg MJ, Plant GW, Pearlman E, Jung S, McMenamin PG: The chemokine receptor CX3CR1 mediates homing of $\mathrm{MHC}$ class II-positive cells to the normal mouse corneal epithelium. Invest Ophthalmol Vis Sci 2007, 48:1568-1574

30. Knickelbein JE, Watkins SC, McMenamin PG, Hendricks RL: Stratification of antigen-presenting cells within the normal cornea. Ophthalmol Eye Dis 2009, 1:45-54

31. Byeseda SE, Burns AR, Dieffenbaugher S, Rumbaut RE, Smith CW, Li Z: ICAM-1 is necessary for epithelial recruitment of gammadelta $T$ cells and efficient corneal wound healing. Am J Pathol 2009, 175: 571-579

32. Bar-On L, Jung S: Defining in vivo dendritic cell functions using CD11c-DTR transgenic mice. Methods Mol Biol 2010, 595:429-442

33. Jung S, Unutmaz D, Wong P, Sano G, De los Santos K, Sparwasser T, Wu S, Vuthoori S, Ko K, Zavala F, Pamer EG, Littman DR, Lang RA: In vivo depletion of $\mathrm{CD} 11 \mathrm{c}+$ dendritic cells abrogates priming of CD8+ T cells by exogenous cell-associated antigens. Immunity 2002, 17:211-220

34. Liu YJ: TSLP in epithelial cell and dendritic cell cross talk. Adv Immunol 2009, 101:1-25

35. Ziegler SF, Artis D: Sensing the outside world: TSLP regulates barrier immunity. Nat Immunol 2010, 11:289-293

36. Gabay C, Lamacchia C, Palmer G: IL-1 pathways in inflammation and human diseases. Nat Rev Rheumatol 2010, 6:232-241

37. Dua HS, Gomes JA, Singh A: Corneal epithelial wound healing. Br J Ophthalmol 1994, 78:401-408

38. Xu K, Yu FS: Impaired epithelial wound healing and EGFR signaling pathways in the corneas of diabetic rats. Invest Ophthalmol Vis Sci 2011, 52:3301-3308

39. Xu KP, Li Y, Ljubimov AV, Yu FS: High glucose suppresses epidermal growth factor receptor/phosphatidylinositol 3-kinase/Akt signaling pathway and attenuates corneal epithelial wound healing. Diabetes 2009, 58:1077-1085

40. Wilson SE, He YG, Weng J, Li Q, McDowall AW, Vital M, Chwang EL: Epithelial injury induces keratocyte apoptosis: hypothesized role for the interleukin-1 system in the modulation of corneal tissue organization and wound healing. Exp Eye Res 1996, 62:325-327

41. Kim WJ, Mohan RR, Wilson SE: Caspase inhibitor z-VAD-FMK inhibits keratocyte apoptosis, but promotes keratocyte necrosis, after corneal epithelial scrape. Exp Eye Res 2000, 71:225-232

42. Zaft T, Sapoznikov A, Krauthgamer R, Littman DR, Jung S: CD11chigh dendritic cell ablation impairs Iymphopenia-driven proliferation of naive and memory CD8+ T cells. J Immunol 2005, 175: 6428-6435
43. Sosnova-Netukova M, Kuchynka P, Forrester JV: The suprabasal layer of corneal epithelial cells represents the major barrier site to the passive movement of small molecules and trafficking leukocytes. $\mathrm{Br} \mathrm{J}$ Ophthalmol 2007, 91:372-378

44. Yamagami S, Yokoo S, Usui T, Yamagami H, Amano S, Ebihara N: Distinct populations of dendritic cells in the normal human donor corneal epithelium. Invest Ophthalmol Vis Sci 2005, 46:4489-4494

45. Yin J, Xu K, Zhang J, Kumar A, Yu FS: Wound-induced ATP release and EGF receptor activation in epithelial cells. J Cell Sci 2007, 120: $815-825$

46. Xu KP, Yin J, Yu FS: Lysophosphatidic acid promoting corneal epithelial wound healing by transactivation of epidermal growth factor receptor. Invest Ophthalmol Vis Sci 2007, 48:636-643

47. Lee JY, Lim YM, Park MJ, Min SY, Cho ML, Sung YC, Park SH, Kim HY, Cho YG: Murine thymic stromal lymphopoietin promotes the differentiation of regulatory T cells from thymic CD4(+)CD8(-)CD25(-) naive cells in a dendritic cell-independent manner. Immunol Cell Biol 2008, 86:206213

48. Park LS, Martin U, Garka K, Gliniak B, Di Santo JP, Muller W, Largaespada DA, Copeland NG, Jenkins NA, Farr AG, Ziegler SF, Morrissey $\mathrm{PJ}$, Paxton $\mathrm{R}$, Sims JE: Cloning of the murine thymic stromal Iymphopoietin (TSLP) receptor: formation of a functional heteromeric complex requires interleukin 7 receptor. J Exp Med 2000, 192:659_ 670

49. Padovan E, Spagnoli GC, Ferrantini M, Heberer M: IFN-alpha2a induces IP-10/CXCL10 and MIG/CXCL9 production in monocyte-derived dendritic cells and enhances their capacity to attract and stimulate CD8+ effector T cells. J Leukoc Biol 2002, 71:669-676

50. Lindell DM, Lane TE, Lukacs NW: CXCL10/CXCR3-mediated responses promote immunity to respiratory syncytial virus infection by augmenting dendritic cell and CD8(+) T cell efficacy. Eur J Immunol 2008, 38:2168-2179

51. Arend WP: The balance between IL-1 and IL-1Ra in disease. Cytokine Growth Factor Rev 2002, 13:323-340

52. Bustin SA, Benes V, Garson JA, Hellemans J, Huggett J, Kubista M, Mueller R, Nolan T, Pfaffl MW, Shipley GL, Vandesompele J, Wittwer CT: The MIQE guidelines: minimum information for publication of quantitative real-time PCR experiments. Clin Chem 2009, 55:611-622

53. Duronio V: The life of a cell: apoptosis regulation by the PI3K/PKB pathway. Biochem J 2008, 415:333-344

54. Erlandsson Harris $\mathrm{H}$, Andersson $U$ : Mini-review: the nuclear protein HMGB1 as a proinflammatory mediator. Eur J Immunol 2004, 34: 1503-1512

55. Lu L: Stress-induced corneal epithelial apoptosis mediated by K+ channel activation. Prog Retin Eye Res 2006, 25:515-538

56. Nishida T, Nakamura M, Mishima H, Otori T, Hikida M: Interleukin 6 facilitates corneal epithelial wound closure in vivo. Arch Ophthalmo 1992, 110:1292-1294

57. Kenchegowda S, Bazan HE: Significance of lipid mediators in corneal injury and repair. J Lipid Res 2010, 51:879-891

58. Crosby LM, Waters CM: Epithelial repair mechanisms in the lung. Am J Physiol Lung Cell Mol Physiol 2010, 298:L715-L731

59. Li Z, Burns AR, Smith CW: Two waves of neutrophil emigration in response to corneal epithelial abrasion: distinct adhesion molecule requirements. Invest Ophthalmol Vis Sci 2006, 47:1947-1955

60. Wilson SE, Mohan RR, Hong J, Lee J, Choi R, Liu JJ: Apoptosis in the cornea in response to epithelial injury: significance to wound healing and dry eye. Adv Exp Med Biol 2002, 506:821-826 